\title{
Evading Vacuum Noise: Wigner Projections or Husimi Samples?
}

\author{
C. R. Müller, ${ }^{1,2}$ C. Peuntinger, ${ }^{1,2,3}$ T. Dirmeier, ${ }^{1,2}$ I. Khan, ${ }^{1,2}$ U. Vogl, ${ }^{1,2}$ Ch. Marquardt,,${ }^{1,2}$ \\ G. Leuchs, ${ }^{1,2}$ L. L. Sánchez-Soto,,${ }^{1,2,4}$ Y. S. Teo, ${ }^{5}$ Z. Hradil, ${ }^{5}$ and J. Řeháček ${ }^{5}$ \\ ${ }^{1}$ Max-Planck-Institut für die Physik des Lichts, Günther-Scharowsky-Straße 1, Bau 24, 91058 Erlangen, Germany \\ ${ }^{2}$ Institut für Optik, Information und Photonik, Universität Erlangen-Nürnberg, Staudtstraße 7/B2, 91058 Erlangen, Germany \\ ${ }^{3}$ Department of Physics, University of Otago, 730 Cumberland Street, Dunedin, New Zealand \\ ${ }^{4}$ Departamento de Óptica, Facultad de Física, Universidad Complutense, 28040 Madrid, Spain \\ ${ }^{5}$ Department of Optics, Palacký University, 17. listopadu 12, 77146 Olomouc, Czech Republic
}

\begin{abstract}
The accuracy in determining the quantum state of a system depends on the type of measurement performed. Homodyne and heterodyne detection are the two main schemes in continuous-variable quantum information. The former leads to a direct reconstruction of the Wigner function of the state, whereas the latter samples its Husimi $Q$ function. We experimentally demonstrate that heterodyne detection outperforms homodyne detection for almost all Gaussian states, the details of which depend on the squeezing strength and thermal noise.
\end{abstract}

PACS numbers: 03.65.Ta, 03.67.Hk, 42.50.Dv, 42.50.Lc

Introduction.- Quantum information has achieved remarkable progress in the past few years and promises even more far-reaching advances in the near future. Pioneering proposals, such as quantum cryptography [1,2] and teleportation [3], just to list the most popular, have been demonstrated in numerous experiments. Furthermore, some of them are already in commercial operation [4].

The key concepts in the field were initially developed mainly for discrete variables, more specifically for qubits. The continuous-variable (CV) approach offers many practical advantages though [5-9]. Here, information is encoded in continuous degrees of freedom; for example, the quadratures of a field mode. Interestingly enough, in this CV setting most protocols can be simply implemented with linear optical components.

Gaussian states constitute a primary tool for CV quantum information processing [10]. They are versatile resources particularly easy to prepare and control. In addition, they are completely characterized by a finite number of parameters (the covariance matrix of the canonical mode operators), despite their infinite-dimensional support. We shall restrict our attention to this set of states.

To capitalize on CV resources, a very efficient detection is paramount. In optical CV implementations, there are two well established schemes. The first one is homodyne detection [11-13], which performs a projective measurement of a rotated field quadrature. This is precisely the marginal distribution of the Wigner function of the state [14], that can thus be efficiently reconstructed [15]. This method has been shown to achieve the ultimate resolution predicted by the Fisher information [16], so it comes as no surprise that it is widely considered to be optimal in the CV community.

The other technique, heterodyne detection [17-27], realizes the approximate measurement of two complementary orthogonal quadratures. This corresponds to a direct sampling of the Husimi $Q$ function [28]. The price to be paid for a simultaneous measurement of noncommuting observables is the presence of additional vacuum noise, as was first pointed out by Arthurs and Kelly [29].
In theory, both the Wigner and Husimi functions are equivalent representations of the state. However, when reconstructed from experimental data, only finite data resources are available. Technical details aside, this fundamental limitation imposes restrictions on the accuracy of the reconstructions.

In this Letter, we make an unbiased assessment of the accuracy of both schemes in realistic scenarios. We shall experimentally corroborate that for almost all Gaussian states, except for states close to the vacuum, heterodyne detection outperforms homodyne detection. We emphasize that this is more than an academic curiosity, for the Wigner function and the Husimi function are a general concept in many fields of physics and our results are of practical relevance in protocols such as CV quantum key distribution [30-32].

Characterizing covariance matrices. - In homodyne detection, one measures the intensities at the outputs of a beam splitter that coherently merges the signal mode and a local oscillator. In this way, data points $x_{\theta}$ are sampled from the marginal distribution of the Wigner function projected along a specific field quadrature at the phase-space angle $\theta$. As we are dealing with Gaussian states, they are fully characterized by the covariance matrix $G_{\mathrm{W}}$ of the Wigner function. We recall that for a vector $\mathbf{Y}=\left(Y_{1} \ldots Y_{n}\right)^{\mathrm{T}}$ of random variables $Y_{j}(\mathrm{~T}$ stands for the transpose), the elements of the covariance matrix are $G_{i j}=\operatorname{Cov}\left(Y_{i}, Y_{j}\right)=\left\langle\left(Y_{i}-\left\langle Y_{i}\right\rangle\right)\left(Y_{j}-\left\langle Y_{j}\right\rangle\right)\right\rangle$. In what follows, we shall set $\langle\mathbf{Y}\rangle=0$, since the independent estimation of a trivial phase-space translation does not change the qualitative trade-off between the two measurement schemes.

Heterodyne detection originally referred to the beating of a signal with a slightly detuned local oscillator. Nowadays, it also comprises two simultaneous homodyne measurements in orthogonal quadratures with a local oscillator of the same frequency: the signal is split by a symmetric beam splitter prior to being projected onto the quadratures $X$ and $P$. In both cases, data gathered for the same phase-space angle $\theta$ are sampled directly from the Husimi $Q$ function, which is the conditional distribution for the pair of complementary quadratures. As the commutation relations are preserved, the split signals are convoluted with the vacuum noise that can be visualized as 


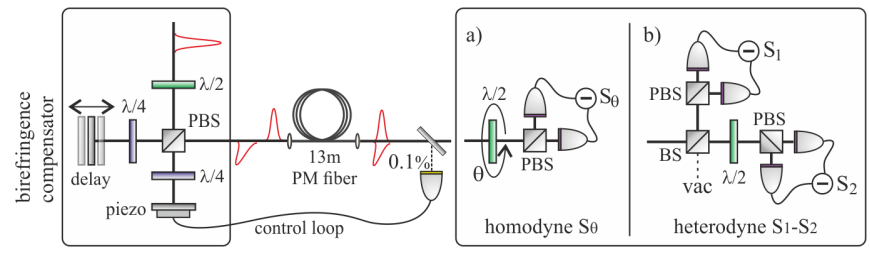

FIG. 1. Sketch of the experimental setup. Pulses from a shot-noise limited laser centered at $1560 \mathrm{~nm}$ are distributed equally on the principal axes of a $13 \mathrm{~m}$ long polarization maintaining fiber. The pulses are individually squeezed due to the Kerr nonlinearity. A birefringent compensator controls the temporal overlap of the emerging pulses and locks them to a relative phase difference of $\pi / 2$, hence forming a $S_{3}$ polarized state. The a) homodyne and b) heterodyne schemes are emulated by Stokes measurements.

entering the unused port of the beam splitter. The covariance matrix for the $Q$ function is then $G_{\mathrm{Q}}=G_{\mathrm{W}}+\mathbb{1}$ (we normalize the variance of the vacuum noise to unity).

Real detectors possess efficiencies $\eta<1$, so that the inferred covariance matrices for the two schemes are respectively [33]

$$
G_{\mathrm{HOM}}=G_{\mathrm{W}}+\frac{1-\eta}{\eta} \mathbb{1}, \quad G_{\mathrm{HET}}=G_{\mathrm{HOM}}+\frac{1}{\eta} \mathbb{1} .
$$

One may confidently make sense of these results from the concepts of marginal and conditional probability distributions. Data sampled according to these two distributions lead to rather different uncertainties. Along the phase-space direction of the unit vector $\mathbf{n}=(\cos \theta \sin \theta)^{\mathrm{T}}$, the marginal variance $\sigma_{\theta}^{2}$ (i.e., homodyne) and conditional variance $\Sigma_{\theta}^{2}$ (i.e., heterodyne) are [34]

$$
\sigma_{\theta}^{2}=\mathbf{n}^{\mathrm{T}} G_{\mathrm{HOM}} \mathbf{n}, \quad \Sigma_{\theta}^{2}=\left(\mathbf{n}^{\mathrm{T}} G_{\mathrm{HET}}^{-1} \mathbf{n}\right)^{-1} .
$$

If the extra noise term $\frac{1}{\eta} \mathbb{1}$ in $G_{\mathrm{HET}}$ were absent, then it is easy to show that $\sigma_{\theta}^{2} \geq \Sigma_{\theta}^{2}$ for any Gaussian state. The equality holds for rotationally symmetric states, as is the case for the vacuum itself.

However, the additional noise $\frac{1}{\eta} \mathbb{1}$ introduces further complexity. For the pertinent example of the vacuum $\left(G_{\mathrm{W}}=\mathbb{1}\right)$, we have now $\sigma_{\theta}^{2}=\frac{1}{\eta} \leq \frac{2}{\eta}=\Sigma_{\theta}^{2}$, so that the uncertainty for data acquired from the marginal distribution (Wigner) is less than those from the conditional distribution (Husimi). Nonetheless, as we shall soon see, for sufficiently bright thermal states, the additional noise in the heterodyne detection becomes negligible, such that, above a threshold thermal photon number, the detrimental impact of the beam splitting noise is overcompensated by the advantage of obtaining two sample points per signal state, ultimately rendering heterodyne detection the superior strategy.

In the same vein, squeezing improves the tomographic performance of heterodyne data over homodyne data, thereby surmounting the intrinsic Arthurs-Kelly measurement uncertainties.

Experimental setup.- With a centered and appropriately oriented coordinate system, the covariance matrix is com-
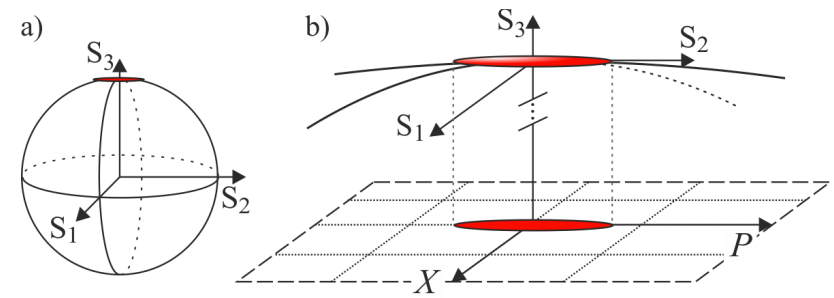

FIG. 2. a) Illustration of the squeezed $S_{3}$-polarized state on the Poincaré sphere. b) Magnification of the polarization state at the pole of the Poincaré sphere. For bright states, the Poincaré sphere has a large radius such that the curvature is locally negligible and the projection in the $S_{1}-S_{2}$ dark plane is equivalent to a rescaled canonical $X-P$ quadrature phase space.

pletely determined by two parameters. A convenient representation in terms of the ellipticity $\lambda$ and phase-insensitive noise $\mu$ yields

$$
G_{\mathrm{W}}=\mu\left(\begin{array}{cc}
1 / \lambda & 0 \\
0 & \lambda
\end{array}\right)
$$

To check the predictions in Ref. [33], we prepared states with different $\lambda$ and $\mu$ parameters employing a fiber-based polarization squeezing setup [35] sketched in Fig. 1. A shotnoise-limited laser (ORIGAMI from Onefive $\mathrm{GmbH}$ ) emitting $220 \mathrm{fs}$ pulses at a repetition rate of $80 \mathrm{MHz}$ and centered at $1560 \mathrm{~nm}$ is coupled equally onto the principal axes of a $13 \mathrm{~m}$-long polarization-maintaining fiber. Quadraturesqueezed states are simultaneously and independently generated in both polarization modes by the Kerr nonlinearity. The strong birefringence of the fiber causes a delay between the emerging quadrature squeezed pulses, which is precompensated with a Michelson-like interferometer placed before the fiber. A weak tap-off measurement $(\approx 0.1 \%)$ at the fiber output is used in a control loop to lock the relative phase between the exiting pulses to $\pi / 2$, so that the light is circularly polarized.

The quantum polarization of light is conveniently described by the Stokes parameters $\mathbf{S}=\left(\begin{array}{lll}S_{1} & S_{2} S_{3}\end{array}\right)^{\mathrm{T}}$. For bright circularly polarized light, as generated in our setup, we have $\left\langle S_{3}\right\rangle=\alpha^{2} \gg 1$, while $\left\langle S_{1,2}\right\rangle=0$. More generally, $\left\langle S_{\theta}\right\rangle=0$ for the rotated version $S_{\theta}=S_{1} \cos \theta+S_{2} \sin \theta$. This defines the "dark plane" - the plane of zero mean intensity. The fluctuations of these operators can then be approximated as [35]

$$
\delta S_{\theta}=\alpha\left(\delta X_{\theta}+\delta X_{\theta+\pi / 2}\right),
$$

where $X_{\theta}$ are the rotated dark-plane quadratures of the bright field [35]. Such measurements are then identical to balanced homodyne detection: the classical excitation is a local oscillator for the orthogonally polarized dark mode, as sketched in Fig. 2. This is a unique feature of polarization measurements and has been used in many experiments [36-40].

Consequently, homodyne tomography is performed by sampling the marginal distributions of the dark plane observables $X_{\theta}$ at 100 equally separated angles $\theta \in[0,2 \pi)$. This is 
done by rotating a half-wave plate positioned after the fiber output with a stepper motor. The heterodyne measurement is realized as simultaneous measurements of conjugate dark plane observables $X_{\theta}$ and $X_{\theta+\pi / 2}$ (which reduce to $X$ and $P$ for $\theta=0$ ).

The two detectors (InGaAs PIN photodiodes, with $98 \%$ quantum efficiency at DC) are balanced and provide a subshot noise resolution in the frequency range between $5 \mathrm{MHz}$ and $30 \mathrm{MHz}$. Each detector has two separate outputs: DC, providing the average values of the photocurrents, and $\mathrm{AC}$, providing the photocurrents amplified in the radio-frequency (RF) spectral range. The RF currents of the photodetectors are mixed with an electronic local oscillator at $12 \mathrm{MHz}$, low-pass filtered (BLP 1.9 with a $-3 \mathrm{~dB}$ cutoff at $2.3 \mathrm{MHz}$ ), amplified (FEMTO DHPVA-100), and digitized by an analog/digital exit converter (Gage CompuScope 1610) at 10 Megasamples per second with a 16-bit resolution and 5 times oversampling.

Data Analysis and Results. - We experimentally prepared a vacuum state and two squeezed states with different degrees of squeezing. We control the squeezing strength as well as the purity of the state by varying the pump power. For the strongly squeezed state, the pulse power at the fiber output was about $55 \mathrm{pJ}$ (i.e., $4.33 \times 10^{8}$ photons/pulse) and a total squeezing strength of $2.51 \mathrm{~dB}$ is observed. In the orthogonal quadrature, the antisqueezing noise is strongly enhanced due to guided acoustic wave Brillouin scattering [41-43] resulting in strongly elliptical states. We observed a noise level of $18.78 \mathrm{~dB}$ above the shot noise level in the antisqueezing direction. For the weakly squeezed state the pulse power was about $40 \mathrm{pJ}$ (i.e., $3.15 \times 10^{8}$ photons/pulse) yielding a squeezing strength of $1.49 \mathrm{~dB}$ and a noise level of $14.62 \mathrm{~dB}$ in the antisqueezing direction. The measurements are performed with different detectors and in a temporally consecutive order. The fluctuations of the observed variances from their average value are within the $1 \%$ margin.

To analyze the performance for noisy symmetric states, we intentionally eliminate the phase information from the squeezed-state data to emulate thermal states. The homodyne data are thermalized by randomly shuffling the data between the 100 measured phase bins, hence tracing out any phase information in the data set. For the heterodyne data, each pair of thermalized quadrature projections $(x, p)_{\text {therm }}$ is derived from the measured quadrature projections $(x, p)_{\mathrm{sqz}}$ of the squeezed states by a random rotation.

In units of coherent shot noise, the thermal states derived from the strongly and weakly squeezed state have a (phaseinvariant) quadrature variance of 38.37 and 15.01, respectively. The variance of a thermal state is directly proportional to the thermal mean photon number, which yields an average thermal photon number of $\left\langle N_{\mathrm{th}}\right\rangle=19.2$ and $\left\langle N_{\mathrm{th}}\right\rangle=7.5$, respectively. The Gaussian phase space contours of the reconstructed states are shown in Fig. 3a).

To quantify the accuracy of the estimated covariance matrix we would need to know the true states. Since this is not strictly feasible, we use instead the average Hilbert-Schmidt distance between the estimation comprising a restricted num-
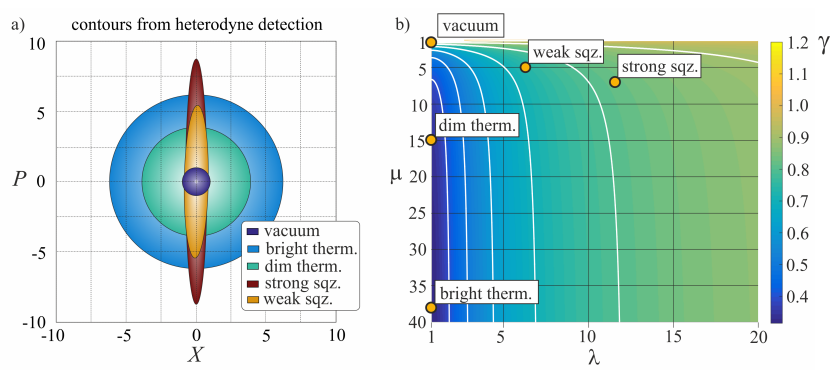

FIG. 3. a) Contours of the reconstructed covariance matrices (shown here for homodyne detection). b) Color-coded contour plot of the $\gamma$ parameter in terms of the ellipticity $\lambda$ and thermal noise $\mu$. The experimentally tested states are indicated by white disks.

ber of samples $G_{\mathrm{W}}$ to the estimation comprising the complete set of acquired data $\widehat{G}_{\mathrm{W}}$ (in our case $10^{6}$ homodyne samples at each of 100 different quadrature angles and $10^{8}$ pairs of heterodyne samples); that is, $\mathbb{D}_{\mathrm{HS}}=\operatorname{Tr}\left\{\left(G_{\mathrm{W}}-\widehat{G}_{\mathrm{W}}\right)^{2}\right\}$. The ratio $\gamma$ between the Hilbert-Schmidt distances yields the relative quality of the reconstructions; viz $\gamma=\mathbb{D}_{\mathrm{HS}}^{(\mathrm{HET})} / \mathbb{D}_{\mathrm{HS}}^{(\mathrm{HOM})}$. This ratio is essentially that of the Cramér-Rao bounds of the two schemes for such a large sample if $\widehat{G}_{\mathrm{W}}$ are maximumlikelihood (ML) estimators [44]. Heterodyne detection is more efficient if $\gamma<1$. In Fig. 3b), we present a $\mu-\lambda$ diagram with both the theoretical predictions and the experimental states.

In the following we do not compensate for the finite efficiency of our detectors, but assume $\eta=1$ for the evaluation of the experimental data. This is a conservative assumption, as the superiority of heterodyne performance even increases for $\eta<1$ [33].

To obtain the correct $\gamma$ values, the reconstructions of covariance matrices from the homodyne tomography data is performed via the Gaussian Maximum Likelihood (ML) algorithm described in Ref. [44]. In this algorithm, the variances of the data collected for fixed quadrature phases are calculated and fed into a recursive loop that optimizes the estimation for the covariance matrix. The results are given in Table I.

For the heterodyne measurement, we directly calculate the sample covariance matrix from the measured joint probability distribution, which is an ML estimator. The data is affected by the electronic noise floor of the detectors, and by background light which we individually record for each measure-

TABLE I. Covariance parameters for both homodyne and heterodyne detection.

\begin{tabular}{lrrrrr}
\hline \hline & \multicolumn{2}{c}{ heterodyne } & & \multicolumn{2}{c}{ homodyne } \\
\cline { 2 - 3 } \cline { 5 - 6 } State & $\mu$ & $\lambda$ & & $\mu$ & \multicolumn{1}{c}{$\lambda$} \\
\hline vacuum state & 0.99 & 1.01 & & 1.01 & 1.00 \\
strongly sqz. & 6.54 & 11.67 & & 6.44 & 11.61 \\
weakly sqz. & 4.62 & 6.29 & & 4.46 & 6.49 \\
bright thermal & 38.36 & 1.00 & & 38.40 & 1.00 \\
dim thermal & 15.01 & 1.00 & & 14.96 & 1.00 \\
\hline \hline
\end{tabular}



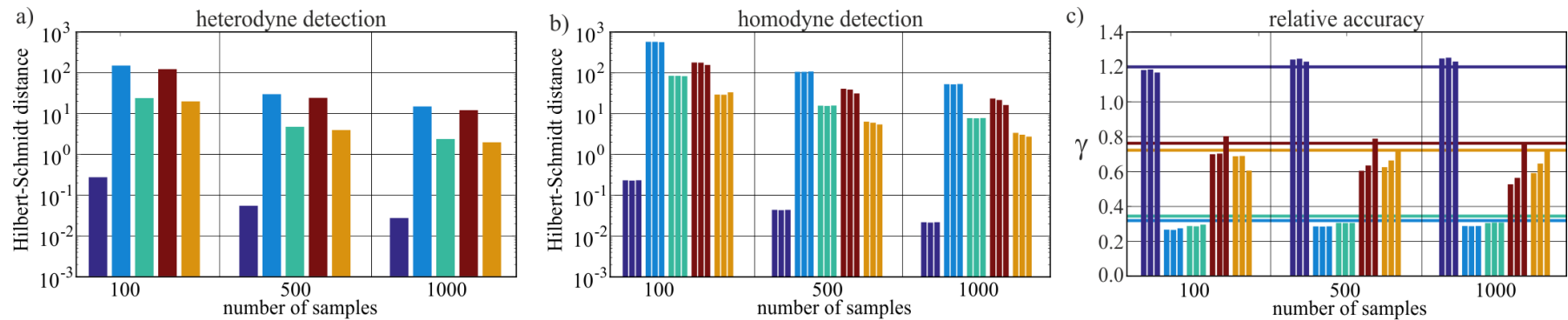

a coherent vacuum $\square$ bright thermal $\square$ weak thermal $\quad$ strongly squeezed $\square$ weakly squeezed

FIG. 4. a) Experimental results for the accuracy of the covariance matrix reconstruction with heterodyne detection. The Hilbert-Schmidt distance is shown for three different numbers of sampling events. b) Experimental results for the accuracy of the covariance matrix reconstruction based on homodyne detection. c) Ratio of the Hilbert-Schmidt distances $\gamma$. The solid lines in the background indicate the theoretical prediction.

ment. We compensate for this additional noise by subtracting its covariance matrix from the measured covariance matrix of the quantum state $G_{\mathrm{HOM} / \mathrm{HET}} \mapsto G_{\mathrm{HOM} / \mathrm{HET}}-G_{\mathrm{ELEC}}$. The covariance matrix of the input state is obtained then as in Eq. (1). The parameters of the states reconstructed by heterodyne detection are also summarized in Table I.

To provide a fair comparison of the covariance matrix reconstructions, the number of sampling events needs to be equal. For the heterodyne detector one sampling event corresponds to a pair of conjugate quadrature projections $(X, P)$, while that from the homodyne detector is given by the single quadrature value $x_{\theta}$ projected onto the field quadrature rotated by an angle $\theta$ with respect to an arbitrary but fixed reference frame. The reference states, against which the accuracy of the covariance matrix estimations are assessed, are reconstructed from extensive measurements comprising $10^{8}$ samples $\left(100 \times 10^{6}\right.$ samples at different quadrature angles for the homodyne detection). Given a fixed number of sampling events, we still have the freedom to choose the number of quadrature phases for the homodyne tomography. We compare the performance for 5, 10, and 15 different angles taken from the 100 angles measured in the extensive reference measurement. To avoid a bias towards a preferred reference frame, these angles are chosen randomly but with constant phase difference between neighboring angles (e.g., $\Delta \theta=2 \pi / 5$ for the 5 angles).

The experimentally observed Hilbert-Schmidt distances for both detection strategies as well as the corresponding $\gamma$ parameter for various sample sizes and for different numbers of measurement angles are shown in Fig. 4. The measured $\gamma$ is in extremely good agreement with the theoretical prediction.

In agreement with theoretical predictions, the HilbertSchmidt distances of both the homodyne and the heterodyne detection decrease by about an order of magnitude when the number of sampling events is increased by a factor of 10 . As could be expected, the estimation accuracy of the phasecovariant states (vacuum, bright/weak thermal) is independent of the number of quadrature angles. For elliptical states, however, the Hilbert-Schmidt distance is initially decreasing with the number of angles, i.e. the estimation gets more accurate by increasing the number of quadrature phases. This is particularly pronounced for the bigger sample sizes, for which the theoretically $\gamma$ parameter is only approached with increasing number of quadrature phases. However, if for a given number of samples too many angles are measured the statistical uncertainty per quadrature is getting worse and the accuracy eventually decreases again.

We also performed Gaussianity tests on the experimental data to check for any non-Gaussian feature. For this, we sort the data into histograms of 101 bins and use the KolmogorovSmirnov test and the $\chi^{2}$ tests, as well as the Kullback-Leibler divergence [45]. We find that all data sets are Gaussian within confidence levels ranging from 95 to $99 \%$.

Concluding remarks. - Apart from a small region of states close to the vacuum state, heterodyne detection outperforms homodyne detection in terms of the reconstruction accuracy for almost all Gaussian states. Therefore, at least for Gaussian states, our direct experimental confirmation of the theory for the performances of the two detection schemes rigorously shows the advantage of the heterodyne detection for quantum tomography. On this note, we believe that this result would be particularly appealing to experimentalists, as the heterodyne detection is conveniently realized without the need for active phase changing elements of the local oscillator beam. Especially in CV quantum key distribution covariance estimation is a crucial part. Moreover, as heterodyne detection directly samples the Husimi $Q$ function, the need for timeconsuming tomographic reconstruction is omitted. The extension of these results to non-Gaussian states is the subject of further research.

Acknowledgments. - We thank Herbert Welling and Sascha Wallentowitz for discussions about different aspects of optical heterodyne detection. We acknowledge financial support from the European Research Council (Advanced Grant PACART), the Spanish MINECO (Grant FIS2015-67963-P), the Technology Agency of the Czech Republic (Grant TE01020229), the Grant Agency of the Czech Republic (Grant No. 15-03194S), and the IGA Project of the Palacký University (Grant No. IGA 
PrF 2016-005).

[1] C. H. Bennett and G. Brassard, "Quantum cryptography: Public key distribution and coin tossing," in Proceedings of the International Conference on Computers, Systems and Signal Processing (IEEE, Bangalore, 1984) pp. 175-179.

[2] A. K. Ekert, "Quantum cryptography based on Bell's theorem," Phys. Rev. Lett. 67, 661-663 (1991).

[3] C. H. Bennett, G Brassard, C. Crépeau, R. Jozsa, A. Peres, and W. K. Wootters, "Teleporting an unknown quantum state via dual classical and Einstein-Podolsky-Rosen channels," Phys. Rev. Lett. 70, 1895-1899 (1993).

[4] "http://www.idquantique.com," .

[5] S. L. Braunstein and P. van Loock, "Quantum information with continuous variables,” Rev. Mod. Phys. 77, 513-577 (2005).

[6] A. Ferraro, S. Olivares, and M. G. A. Paris, Gaussian States in Continuous Variable Quantum Information (Bibliopolis, Napoli, 2005).

[7] N. Cerf, G. Leuchs, and E. S. Polzik, eds., Quantum Information with Continuous Variables of Atoms and Light (Imperial College Press, London, 2007).

[8] U. L. Andersen, G. Leuchs, and C. Silberhorn, "Continuousvariable quantum information processing," Laser Photonics Rev. 4, 337-354 (2010).

[9] G. Adesso, S. Ragy, and A. R. Lee, "Continuous variable quantum information: Gaussian states and beyond," Open Syst. Inf. Dyn. 21, 1440001 (2014).

[10] C. Weedbrook, S. Pirandola, R. García-Patrón, N. J. Cerf, T. C. Ralph, J. H. Shapiro, and S. Lloyd, "Gaussian quantum information,” Rev. Mod. Phys. 84, 621-669 (2012).

[11] H. P. Yuen and V. W. S. Chan, "Noise in homodyne and heterodyne detection," Opt. Lett. 8, 177-179 (1983).

[12] G. L. Abbas, V. W. S. Chan, and T. K. Yee, "Local-oscillator excess-noise suppression for homodyne and heterodyne detection," Opt. Lett. 8, 419-421 (1983).

[13] B. L. Schumaker, "Noise in homodyne detection," Opt. Lett. 9 , 189-191 (1984).

[14] K. Vogel and H. Risken, "Determination of quasiprobability distributions in terms of probability distributions for the rotated quadrature phase," Phys. Rev. A 40, 2847-2849 (1989).

[15] A. I. Lvovsky and M. G. Raymer, "Continuous-variable optical quantum-state tomography,” Rev. Mod. Phys. 81, 299-322 (2009).

[16] Z. Hradil, R. Myška, T. Opatrný, and J. Bajer, "Entropy of phase measurement: Quantum phase via quadrature measurement," Phys. Rev. A 53, 3738-3742 (1996).

[17] A. Javan, E. A. Ballik, and W. L. Bond, "Frequency characteristics of a continuous-wave He-Ne optical maser," J. Opt. Soc. Am. 52, 96-98 (1962).

[18] W. S. Read and R. G. Turner, "Tracking heterodyne detection," Appl. Opt. 4, 1570-1573 (1965).

[19] H. R. Carleton and W. T. Maloney, "A balanced optical heterodyne detector," Appl. Opt. 7, 1241-1243 (1968).

[20] H. Gerhardt, H. Welling, and A. Güttner, "Measurements of the laser linewidth due to quantum phase and quantum amplitude noise above and below threshold. I," Z. Phys. 253, 113-126 (1972).

[21] H. Yuen and J. H. Shapiro, "Optical communication with twophoton coherent states-part III: Quantum measurements realizable with photoemissive detectors," IEEE Trans. Inform. The- ory IT26, 78-92 (1980).

[22] J. H. Shapiro and S. Wagner, "Phase and amplitude uncertainties in heterodyne detection," IEEE J. Quantum Electron. QE20, 803-813 (1984).

[23] J. Shapiro, "Quantum noise and excess noise in optical homodyne and heterodyne receivers," IEEE J. Quantum Electron. QE21, 237-250 (1985).

[24] N. G. Walker and J. E. Carroll, "Multiport homodyne detection near the quantum noise limit," Opt. Quantum Electron. 18, 355363 (1986).

[25] M. J. Collett, R. Loudon, and C. W. Gardiner, "Quantum theory of optical homodyne and heterodyne detection," J. Mod. Opt. 34, 881-902 (1987).

[26] Y. Lai and H. A. Haus, "Characteristic functions and quantum measurements of optical observables," Quantum Opt. 1, 99-116 (1989).

[27] G. M. D'Ariano, "Homodyning as universal detection," in Quantum Communication, Computing, and Measurement, edited by O. Hirota, A. S. Holevo, and C. M. Caves (Springer US, Boston, MA, 1997) pp. 253-264.

[28] S. Stenholm, "Simultaneous measurement of conjugate variables," Ann. Phys. 218, 233-254 (1992).

[29] E. Arthurs and J. L. Kelly, "On the simultaneous measurement of a pair of conjugate observables," Bell Syst. Tech. J. 44, 725729 (1965).

[30] S. Lorenz, N. Korolkova, and G. Leuchs, "Continuous-variable quantum key distribution using polarization encoding and post selection,” Appl. Phys. B 79, 273-277 (2004).

[31] A. M. Lance, T. Symul, V. Sharma, C. Weedbrook, T. C. Ralph, and P. K. Lam, "No-switching quantum key distribution using broadband modulated coherent light," Phys. Rev. Lett. 95, 180503 (2005).

[32] V. Scarani, H. Bechmann-Pasquinucci, N. J. Cerf, M. Dušek, N. Lütkenhaus, and M. Peev, "The security of practical quantum key distribution,” Rev. Mod. Phys. 81, 1301-1350 (2009).

[33] J. Řeháček, Y. S. Teo, Z. Hradil, and S. Wallentowitz, "Surmounting intrinsic quantum-measurement uncertainties in Gaussian-state tomography with quadrature squeezing," Sci. Rep. 5, 12289 (2015).

[34] D. R. Cox, Principles of Statistical Inference (Cambridge University Press, Cambridge, 2006).

[35] J. Heersink, V. Josse, G. Leuchs, and U. L. Andersen, "Efficient polarization squeezing in optical fibers," Opt. Lett. 30, 11921194 (2005).

[36] P. Grangier, R. E. Slusher, B. Yurke, and A. LaPorta, "Squeezed-light-enhanced polarization interferometer," Phys. Rev. Lett. 59, 2153-2156 (1987).

[37] V. Josse, A. Dantan, A. Bramati, and E. Giacobino, "Entanglement and squeezing in a two-mode system: theory and experiment," J. Opt. B 6, S532-S543 (2004).

[38] Ch. Marquardt, J. Heersink, R. Dong, M. V. Chekhova, A. B. Klimov, L. L. Sánchez-Soto, U. L. Andersen, and G. Leuchs, "Quantum reconstruction of an intense polarization squeezed optical state,” Phys. Rev. Lett. 99, 220401 (2007).

[39] C. R. Müller, B. Stoklasa, C. Peuntinger, C. Gabriel, J. Řeháček, Z. Hradil, A. B. Klimov, G. Leuchs, Ch. Marquardt, and L. L. Sánchez-Soto, "Quantum polarization tomography of bright squeezed light,” New J. Phys. 14, 085002 (2012).

[40] C. Peuntinger, B. Heim, C. R. Müller, C. Gabriel, Ch. Marquardt, and G. Leuchs, "Distribution of squeezed states through an atmospheric channel," Phys. Rev. Lett. 113, 060502 (2014).

[41] R. M. Shelby, M. D. Levenson, and P. W. Bayer, "Guided acoustic-wave Brillouin scattering," Phys. Rev. B 31, 5244 5252 (1985). 
[42] R. M. Shelby, M. D. Levenson, S. H. Perlmutter, R. G. DeVoe, and D. F. Walls, "Broad-band parametric deamplification of quantum noise in an optical fiber,' Phys. Rev. Lett. 57, 691694 (1986).

[43] D. Elser, U. L. Andersen, A. Korn, O. Glöckl, S. Lorenz, Ch. Marquardt, and G. Leuchs, "Reduction of guided acoustic wave Brillouin scattering in photonic crystal fibers," Phys. Rev. Lett. 97, 133901 (2006).
[44] J. Řeháček, S. Olivares, D. Mogilevtsev, Z. Hradil, M. G. A. Paris, S. Fornaro, V. D'Auria, A. Porzio, and S. Solimeno, "Effective method to estimate multidimensional Gaussian states," Phys. Rev. A 79, 032111 (2009).

[45] H. C. Thode, Testing for Normality (Marcel Dekker, New York, 2002). 